\title{
ACERCA DEL LECTOTIPO DE BacCHARIS CAPRARIIFolia (AsteraceAe, Astereae)
}

\author{
DANIEL A. GIULIANO ${ }^{1}$
}

Resumen: Se corrige la citación del lectotipo para Baccharis caprariifolia DC.

Palabras clave: Baccharis caprariifolia, Asteraceae, lectotipo.

Summary: On the lectotype of Baccharis caprariifolia (Asteraceae, Astereae). The citation of the lectotype of Baccharis caprariifolia DC. is corrected.

Key words: Baccharis caprariifolia, Asteraceae, lectotype.

\section{INTRODUCCIÓN}

La especie Baccharis caprariifolia DC. (Asteraceae, Astereae) fue originalmente descripta por el célebre botánico Augustin Pyramus de Candolle en base a dos ejemplares colectados por el botánico y naturalista Friedrich Sellow -d488 y s.n.-, consignados bajo los números 966 y 968 respectivamente del ex Herbarium Imperialis Brasiliensis (H.I.B.).

Debido a un error involuntario, en el tratamiento del género Baccharis para la Flora Argentina (Giuliano \& Plos, 2014), se designó como lectotipo de esta especie el ejemplar F. Sellow s.n. (= H.I.B. 975), P 00755497; si bien el número de la hoja correspondiente al Herbario del Museo de Historia Natural de París es el correcto, el número del Herbarium Imperialis Brasiliensis corresponde al lectotipo de Baccharis cognata DC. (Giuliano \& Plos, 2014: 65).

En consecuencia, se corrige aquí la citación del lectotipo de Baccharis caprariifolia.

\footnotetext{
1 Área de Botánica, Facultad de Ciencias Agrarias y Forestales, Universidad Nacional de La Plata, Av. 60 y 119, C.C. 31, 1900 La Plata, Argentina; giuliano@fcnym. unlp.edu.ar
}

Baccharis caprariifolia DC., Prodr. 5: 416. 1836. Tipo. Brasil. Rio Grande do Sul, sin localidad consignada, 1833, F. Sellow s.n. (= H.I.B. 968) (lectotipo, P 00755497!, aquí designado; isolectotipos, G-DC, R 000038135!).

Este ejemplar es seleccionado como lectotipo entre los especímenes depositados en $\mathrm{P}$ debido a que presenta dos plantas que portan numerosos capítulos, mientras que el ejemplar que representa al otro sintipo consiste solamente en una planta en el estado vegetativo.

Cabe mencionar aquí que en el tratamiento del género Baccharis para la Flora Argentina (Giuliano \& Plos, 2014) no se han considerado las indicaciones de tipos efectuadas por Malagarriga Heras (1958) como designaciones de lectotipos (cfr. Heiden \& Pirani, 2012: 43, 46; Müller, 2013), puesto que este mismo autor menciona claramente en el final de la introducción de su trabajo (Malagarriga Heras, 1958: 276) que su intención al realizar estas anotaciones consiste solamente en indicar cuáles de los ejemplares correspondientes a especies de la subtribu Baccharidinae por él examinados en el Herbario P constituyen tipos en sentido genérico, sin otorgarles especificación alguna; el autor aclara asimismo que en su opinión "es necesario conocer el material existente en los grandes herbarios antes 
de especificar" la naturaleza del tipo. En el caso de Baccharis caprariifolia, Malagarriga Heras (1958: 280) colocó la indicación "type" sólo al ejemplar F. Sellow d488 (= H.I.B. 966), omitiendo anotar dicha observación para el ejemplar F. Sellow s.n. (= H.I.B. 968), lo cual fue interpretado por Müller (2013) como una designación de lectotipo por parte de aquel autor.

Se considera aquí que el Art. 9.9 del International Code of Nomenclature (McNeill et al., 2012) no es aplicable a estos casos, ya que el mismo se refiere a errores a ser corregidos debido a un uso equivocado de los términos que denotan tipos, mientras que como se acaba de mencionar, Malagarriga Heras deliberadamente no tuvo la intención de especificar la clase de tipos por él examinados.

Asimismo vale aclarar que por un error involuntario en la citación de la sigla del autor, a lo largo del tratamiento del género Baccharis para la Flora Argentina, en aquellos nombres de taxones donde se consigna "I.L. Teodoro Malag.", debe decir solamente "Malag."; Irmão Teodoro Luis era el nombre religioso adoptado por Ramón de Peñafort Malagarriga Heras, el cual empleó en sus publicaciones hasta principios de la década de 1970.

\section{Agradecimientos}

A D. J. Nicholas Hind por sus consideraciones acerca del carácter de las indicaciones de tipos realizadas por Ramón de Peñafort Malagarriga Heras en ejemplares correspondientes a especies de la subtribu Baccharidinae depositados en P.

\section{Bibliografía}

GIULIANO, D. A. \& A. PLOS. 2014. Baccharis. En: ZULOAGA, F. O., M. J. BELGRANO \& A. M. ANTON (eds.), FREIRE, S. E. (coord.), Flora Argentina: flora vascular de la República Argentina. Dicotyledoneae, Asteraceae, Vol. 7, Tomo 1: 43123. Instituto de Botánica Darwinion, CONICET, San Isidro.

HEIDEN, G. \& J. R. PIRANI. 2012. A synopsis and notes for Baccharis subgen. Tarchonanthoides (Asteraceae: Astereae). Phytotaxa 60: 41-49.

MALAGARRIGA HERAS, R. DE P. [IRMÃO TEODORO LUIS]. 1958. Notes critiques à propos des Baccharidinae de l'Herbier du Laboratoire de Phanérogamie du Muséum d'Histoire Naturelle de Paris. Notul. Syst. 15: 275-298.

McNEILL, J., F. R. BARRIE, W. R. BUCK, V. DEMOULIN, W. GREUTER, D. L. HAWKSWORTH, P. S. HERENDEEN, S. KNAPP, K. MARHOLD, J. PRADO, W. F. PRUD'HOMME VAN REINE, G. F. SMITH, J. H. WIERSEMA \& N. J. TURLAND (eds.). 2012. International Code of Nomenclature for algae, fungi and plants (Melbourne Code), adopted by the Eighteenth International Botanical Congress Melbourne, Australia, July 2011. Regnum Vegetabile 154. Koeltz Scientific Books, Koenigstein.

MÜLLER, J. 2013. World checklist of Baccharis L. (Compositae-Astereae). Version 2013-09-03. Disponible en: http://www.spezbot.uni-jena.de/ wp-content/uploads/2013/09/World-checklist-ofBaccharis-L [Acceso: 15 Septiembre 2014].

Recibido el 8 de octubre de 2014, aceptado el 15 de octubre de 2014. 